Cristina Polo Usaola, Marisa López Gironés, Daniel Olivares Zarco, Antonio Escudero Nafs, Beatriz Rodríguez Vega, Alberto Fernández Liria

\title{
AUTOINCULPACIÓN EN MUJERES QUE SUFREN MALTRATO POR PARTE DE SU PAREJA. FACTORES IMPLICADOS
}

\author{
SELFBLAME IN WOMEN BATTERED BY THEIR PARTNERS. IMPLIED FACTORS
}

\section{RESUMEN}

Este trabajo tiene como objetivos estudiar el tipo de atribución de responsabilidad que realizan las mujeres que sufren maltrato físico por parte de su pareja y la relación entre el tipo de atribución con otros tipos de abuso en la infancia y otras variables. La muestra incluye 134 mujeres consultantes de tres Centros de salud Mental en las que se detectó abuso físico de su pareja. Los resultados señalan que un $37,3 \%$ pensaban que ellas habían sido fundamentalmente las responsables del abuso; $47,6 \%$ pensaban que la responsabilidad era fundamentalmente de sus parejas y $15,1 \%$ que era una responsabilidad compartida. Dentro de las que se autoinculparon, $32,6 \%$ pensaban que la culpa era de tipo caracterial y $67,4 \%$ conductual. Este grupo presentaba más antecedentes de intentos de suicidio, mayor índice de separaciones en la infancia y mayor frecuencia de abuso físico infantil. En análisis multivariante con regresión logística la única variable predictora fue haber sufrido maltrato del padre en la infancia. Se discuten otros hallazgos.

Palabras clave: mujeres maltratadas, atribución, autoinculpación, maltrato infantil.

\section{ABSTRACT}

The objective is to study the type of attribution of responsibility with women battered by their partners, and the relationship between the type of attribution with other types of abuse in infancy. The sample includes 134 women attending three Mental Health Centers in whom physical abuse by their partners was detected using specific questionnaires. Results: We detected that $37,3 \%$ of women though they were mainly responsible; $47.6 \%$ though the responsibility was basically their partners and $15.1 \%$ though it was a shared responsibility. Within the women that blamed themselves, $32.6 \%$ believed their character was to blame and $67.4 \%$ thought it because of their behavior. Women that blamed themselves had the following characteristics: more suicide attempts, greater number of divorces in infancy and a higher frequency of physical abuse in infancy. The only predictive variable with multivariate analysis with logistic regression, was having suffered abuse by the father during infancy. Other findings are discussed.

Key words: battered women, attribution, self-blame, childhood abuse, health mental center. 


\section{INTRODUCCIÓN}

El maltrato a la mujer por parte de su pareja es un tema de interés creciente en el mundo sanitario. Son ampliamente conocidas las consecuencias para la salud física y psíquica de la mujer y su entorno familiar que este fenómeno tiene. En nuestro medio se han descrito diversas herramientas útiles para la evaluación psicológica y el abordaje de la mujer maltratada $(1,2)$. En la práctica clínica, una pregunta frecuente entre los profesionales que trabajamos en este campo tiene que ver con el mantenimiento del maltrato. Para explicar este hecho se han formulado distintas teorías. Lorente Acosta (3) afirma que hay que conocer las características del síndrome de agresión a la mujer para poder comprender los efectos y causas del mismo y ver así las diferencias con otras situaciones de violencia interpersonal. En este sentido tiene especial interés el estudio del ya muy conocido «ciclo de la violencia». El hecho de que tras el episodio de agresión suceda la llamada «fase de luna de miel», causa un efecto descrito como especialmente seductor en una mujer que habitualmente carece de apoyos. A medida que la agresión va quedando atrás, las explicaciones del hombre y las justificaciones de la mujer van tomando fuerza.

La teoría de la atribución, clásicamente usada en la depresión $(4,5)$, también se ha utilizado para intentar ayudar a esclarecer este fenómeno. De acuerdo con esta teoría, los seres humanos tenemos la necesidad de atribuir un significado a lo que nos sucede con el fin de poder tener un control sobre ello. La necesidad de explicaciones causales surge, sobre todo, si ocurren acontecimientos traumáticos, como es el caso de personas victimas de accidentes, agresiones, enfermedades graves o incurables, etc (6). Estas atribuciones influyen en cómo las personas sienten, piensan y se comportan. Se ha argumentado que determinadas formas de atribución conducen con más facilidad al ajuste personal y social en situaciones de adversidad. Por ejemplo, en el clásico estudio realizado por Bulman y Wortman (7) en el que se estudiaron a víctimas de accidentes con graves lesiones medulares, se observó que tenían mejor ajuste los pacientes que tendían a culparse a ellos mismos por el accidente y los que se consideraban menos capaces de prevenirlo. En otro estudio, Brewin (6) estudió las atribuciones de pacientes que habían tenido accidentes de trabajo y habían tenido como consecuencia fracturas leves. Las personas que tuvieron mejor pronóstico y se incorporaron antes al trabajo fueron aquellas que se sintieron responsables del accidente.

Entre los investigadores de situaciones de violencia, el estudio de la autoinculpación también ha tenido un interés considerable. En casos de violación, se ha observado que la autoinculpación es una reacción común (8); sin embargo, en los estudios realizados sobre procesos atribucionales en violencia a la mujer por parte de su pareja, los resultados no son uniformes y la incidencia de autoinculpación observada es variable. Se ha sugerido que la alta incidencia de autoinculpación en las víctimas de violación puede deberse al hecho de que suele ocurrir solamente una vez. En el 
caso de la violencia en la pareja, la violencia tiende a repetirse y esto puede conducir a cambios en las atribuciones de culpa a lo largo del tiempo. Así, los sentimientos de autoinculpación serían más frecuentes al inicio de la relación (9).

Hay varias teorías que intentan explicar el fenómeno de la autoinculpación en el maltrato(10): Algunos autores explican que ésta refleja cómo internalizan las víctimas las reacciones negativas, que han sido expresadas por la sociedad con relación a estos fenómenos. También se ha argumentado que en el tipo de atribución influye que el abuso ocurre en el contexto de una explotación psicológica, donde los abusadores usan su poder para manipular la percepción de realidad de las víctimas. Es posible que un soporte social positivo ayude a cambiar estas percepciones. Si comparamos con víctimas de otros tipos, las investigaciones hechas en víctimas de crímenes violentos o de violaciones, aportan resultados en los que la mayoría de las mujeres tienden a culparse a sí mismas de su victimización. Esta tendencia se piensa que es un mecanismo que contribuye al ajuste positivo de las víctimas para restaurar su sensación de control sobre el entorno (11). En el caso de las mujeres maltratadas por su pareja, las características son distintas: hay que recordar que la agresión se produce en el seno de una relación de pareja, en muchas ocasiones no se limita a un solo episodio y tiene dinámicas particulares y diferentes a las que se producen en violencias efectuadas por extraños.

Algunos autores (8) distinguen dos tipos de autoinculpación: «conductual» y «caracterial». La primera hace relación a la culpa que aparece cuando una persona siente que son sus conductas, los actos que realiza o los que omite, los que producen el acto violento. El tipo de culpa «caracterial» hace referencia al fenómeno que aparece cuando las víctimas se sienten culpables por su forma de ser, por rasgos de su carácter. Es un tipo de culpa más relacionado con la autoestima. En el caso de las víctimas de violación, la culpa atribuida a la conducta es más frecuente. En un conocido estudio hecho entre 335 mujeres, atendidas en centros de asistencia a mujeres violadas, se encontró que el porcentaje de mujeres que se sentían culpables por haber sido violadas era de un $74 \%$. De ellas, el $69 \%$ atribuían la culpa a su comportamiento y el $19 \%$ a su carácter (8). Uno de los motivos que se ha argumentado para el hecho de que las víctimas de violación presenten un sentimiento de culpa relacionado con su conducta es, que este tipo de culpabilización suele surgir cuando los hechos ocurren en una sola ocasión. Como ya se expuso anteriormente, este motivo también se ha argumentado para justificar que la culpa sea más frecuente en víctimas de violación que en maltrato. Parece que el ser objeto de violencia de modo reiterado está relacionado con la autoinculpación atribuida al propio carácter.

Desde otra perspectiva, Eisikovits (12) en un trabajo realizado con metodología cualitativa, señala que existen dos direcciones distintas en la investigación de sentimientos de culpa en situaciones de maltrato. La primera intenta diferenciar -desde 
un punto de vista fenomenológico y teórico- entre culpa y vergüenza. Considera que la culpa está más orientada hacia conductas específicas y que necesita reparación, mientras que la vergüenza es un sentimiento más generalizado, menos limitado a situaciones concretas y más orientado hacia uno mismo «como un todo». La segunda aproximación ignora la distinción entre verguenza y culpa. Esta perspectiva señala que es difícil definir la culpa apartada de la vergüenza porque a menudo se solapan y las personas tienden a experimentarlas concomitantemente.

Centrándonos en nuestro estudio, nuestro interés es estudiar el tipo de atribución causal, especialmente autoinculpación, que la mujer realiza cuando es víctima de una relación violenta por parte de su pareja y la relación entre el tipo de atribución con algunas experiencias relacionales infantiles y características clínicas. Existen numerosos trabajos que encuentran relación entre haber sufrido abuso sexual en la infancia y sufrir en la edad adulta mayor frecuencia de agresiones sexuales y agresiones físicas de la pareja (13-20). Sin embargo, son escasos los estudios y no conocemos ninguno en nuestro medio, que estudien la relación entre el tipo de atribución y haber sufrido abuso en la infancia.

Partimos de la idea de que haber sufrido experiencias infantiles de abuso supone un factor de riesgo para que las mujeres que sufran abuso físico en edad adulta se culpabilicen por el mismo. Es conocido que cuando un niño sufre agresiones de alguien a quien ama, una posible organización de estas experiencias es la negación del daño o la autoculpabilización por la agresión. Este sentimiento de culpa ante los abusos sufridos puede acompañar a estas personas a lo largo de su existencia, y provocar que se justifiquen las agresiones de los demás (21). Nos interesa también si determinadas características de los agresores, estudiadas desde la perspectiva de las mujeres, influyen en el tipo de atribución que ellas realizan. Aunque no hemos encontrado estudios en esta línea, pensamos que algunos antecedentes de los agresores pueden influir en que la mujer tienda a desresponsabilizarles de su agresión.

Teniendo en cuenta que el trabajo que estamos realizando transcurre en un medio clínico, nuestro interés también incluía estudiar la relación entre el tipo de atribución y algunas características clínicas. En algunos estudios se ha observado que las mujeres que se culpan por una relación violenta, presentan con mayor frecuencia diagnóstico de depresión (22).

Considerando estos antecedentes, los OBJETIVOS de nuestro estudio son:

- Estudiar el tipo de atribución de responsabilidad que realizan frente al abuso sufrido por parte de su pareja un grupo de mujeres consultantes de servicios de salud mental.

- Relacionar el tipo de atribución con experiencias infantiles de abuso: haber sufrido abuso físico por parte de los padres, haber presenciado abuso físico entre los padres y haber sufrido abuso sexual en la infancia. 
- Estudiar si existe asociación entre el tipo de atribución que hacen las mujeres con algunas características de sus parejas estudiadas a través de la perspectiva de ellas: haber sufrido abuso físico y/o sexual en la infancia, haber presenciado maltrato entre los padres, tener problemas relacionados con el alcohol y tener antecedentes de tratamiento psiquiátrico.

- Investigar si el tipo de atribución que realizan tiene que ver con algunas características clínicas: diagnóstico e intentos autolíticos.

\section{MÉTODO}

\section{a) Participantes y criterios de inclusión}

Consideramos una muestra compuesta por 134 mujeres con las siguientes características:

- Mayores de 18 años.

- Que hubieran mantenido o mantuvieran una relación de convivencia con una pareja.

- Que asistieron a las consultas ambulatorias de cuatro profesionales incluidos en el estudio, en tres Centros de Salud Mental del Área 4 de la Comunidad de Madrid (Hortaleza, Ciudad Lineal y Fuencarral) durante un año.

- En las que se detectó que habían sufrido maltrato físico por parte de su pareja, después de haber realizado las escalas específicas para la detección del mismo («Conflict Tactics Scale»)(23,24).

Se incluyeron todos los diagnósticos de la clasificación CIE-10, excepto los señalados como criterios de exclusión: Se excluyeron aquellas mujeres que presentaron según la CIE-10 diagnóstico de Trastorno mental orgánico (F00-F09); Esquizofrenia, trastorno esquizotípico y trastorno por ideas delirantes(F20- F29); Episodio depresivo grave con y sin síntomas psicóticos (F31.4, F31.5, F32.2,F32.3, F33.2, F33.3); Episodio maniaco. (F30, F31.0, F31.1, F31.2, F31.6). En los dos últimos casos (episodio depresivo grave y episodio maníaco) mantuvimos la entrevista cuando los síntomas psicóticos y los maniformes habían remitido y la intensidad de los síntomas depresivos había disminuido. Excluimos estos diagnósticos intentando evitar que la respuesta a los cuestionarios no se alterara por la existencia de déficits cognitivos significativos, síntomas psicóticos o alteraciones severas del estado de ánimo.

\section{b) Instrumentos de medida:}

Se realizó un cuestionario específico para esta investigación que recogía:

- Datos de las mujeres:

- Sociodemográficos: edad, estado civil, nivel educativo, profesión, situación laboral y tipo de convivencia. 
- Datos de la infancia: Existencia de acontecimientos vitales de: pérdida-entendida como la muerte- o separación de uno o ambos padres antes de los 17 años.

- Datos clínicos: diagnóstico clínico (Según la CIE-10)(OMS, 1992) e intentos de suicidio.

- Otras variables: consecuencias físicas del maltrato, interposición de denuncias, intensidad del maltrato, duración del mismo.

Datos de los agresores (recogidos a partir de información de la mujer):

- Sociodemográficos: Nivel educativo, profesión y situación laboral.

- Antecedentes de tratamiento psiquiátrico.

- Problemas relacionados con consumo de alcohol o de otros tóxicos. (Se preguntaba a la mujer si pensaba que su pareja tenía problemas relacionados con el abuso de alcohol u otros tóxicos).

- Conocimiento de existencia de agresiones físicas entre sus padres y conocimiento de existencia de abuso físico y/o sexual en la infancia.

- ESCALAS DE «TÁCTICAS DE CONFLICTO» (CTS) $(23,24)$. Se usaron para detectar:

- la existencia de violencia física por parte de la pareja en el último año y a lo largo de toda la relación;

- el recuerdo de haber presenciado violencia física entre los padres, antes de los 16 años de edad y

- el hecho de haber sufrido violencia física, por parte de sus padres, antes de los 16 años de edad.

Miden frecuencia e intensidad. Este instrumento es el más ampliamente utilizado para detectar violencia en la relación de pareja. Existe un manual con más de 200 referencias a la validez y fiabilidad del CTS. (25). La versión que utilizamos en castellano es la forma «R», traducida por Etiony Aldarondo en 1992 (25). En este cuestionario, se pide a los sujetos que indiquen si han usado ciertas conductas para resolver conflictos en sus relaciones íntimas y la frecuencia de su utilización. Las opciones de frecuencia son $0=$ nunca; $1=$ una vez; $2=$ dos veces; $3=$ de tres a cinco veces; $4=$ de seis a diez veces; $5=$ de 11 a 20 veces; $6=$ más de 20 veces. Dentro de esta escala nosotros usamos la subescala «agresión verbal», la subescala «violencia física moderada» y la subescala «violencia física grave». Este uso parcial está autorizado por el autor de la escala (24). Los items que el autor incluye en la subescala «agresión verbal» son: «amenazó con darle o tirarle algo» y «tiró, estrelló, golpeó o pateó alguna cosa». En la subescala «violencia física moderada» incluye los items: «le tiró algo», «le empujó, agarró ó le echó», «le dió con la mano», 
«le pateó, mordió o le pegó con el puño» y «le pegó o trató de pegarle con alguna cosa». En la subescala «violencia física severa» incluye: «le dió una paliza», «le agarró por el cuello», le amenazó con cuchillo o pistola» y «usó cuchillo o pistola».

- CUESTIONARIO DE DETECCión DE ABUSO SEXUAL INFANTIL (26) Según este cuestionario se consideran «experiencias de abuso sexual» aquellas relaciones de contenido sexual en las que había habido algún contacto corporal con alguien mayor de 16 años o para edades entre los 13 y los 15 años con alguien al menos cinco años mayor. Es el instrumento más utilizado para detección de abuso sexual infantil.

- EVALUACIÓN DE ATRIBUCIÓN DE RESPONSABILIDADES POR LA VIOLENCIA (9). Se trata de un cuestionario adaptado por Andrews y Brewin (9) a partir de la clasificación que realizó Janoff-Bulman (8) sobre atribuciones de culpa en situaciones de violencia. En este cuestionario se pregunta a la mujer si a lo largo de la relación de maltrato, consideraba que era ella responsable o culpable de la violencia en la relación o si consideraba que esta responsabilidad se relacionaba con su pareja o con ambos. La pregunta formulada es: «¿Hasta que punto se siente o se sentía usted responsable o, de algún modo, culpable de la violencia física de su pareja? ¿Hasta que punto piensa o pensaba que era su pareja la culpable de esa violencia?». La escala de respuestas es de 3 opciones: «Fundamentalmente yo soy/era la responsable; «Fundamentalmente mi pareja es/era la responsable»; «Ambos somos/éramos igualmente responsables».

Posteriormente se realizan preguntas para diferenciar entre culpabilidad de tipo caracterial o conductual. Se entiende por «culpa caracterial» la que identifica una cualidad estable en uno mismo que sigue a un suceso negativo (ser incompetente, inferior, patológico...). La «culpa conductual» se relaciona con acciones específicas controlables y puntuales que conducen a sucesos negativos (haber contestado mal, justificar el incidente por tener presiones en el trabajo, etc)(8). Después de explicar lo que significaban estos conceptos, intentábamos que las mujeres pusieran ejemplos que sirvieran para clasificar la culpa (de ellas, en el caso de que se hubieran autoinculpado o de los agresores, en el caso de que los responsabilizaran). La escala de respuestas era de dos opciones: caracterial y conductual. Con el fin de controlar la posibilidad de sesgos, se transcribieron los ejemplos que las mujeres habían puesto para definir si la atribución era de tipo caracterial o conductual. La inclusión de las respuestas en los distintos tipos de culpa se revisó de forma conjunta entre los cuatro evaluadores, obteniéndose acuerdos en todos los casos. 


\section{c) Análisis de los datos}

El análisis estadístico fue llevado a cabo usando programas estándar (Statistical Package for the Social Sciences). El conjunto de datos se procesó utilizando la versión 9.0 del paquete estadístico SPSS/PC+. En un primer lugar, se analizó la muestra con fines puramente descriptivos. Utilizamos la media y la desviación típica para los datos cuantitativos. Los cualitativos están expresados en forma de porcentaje. Posteriormente se realizó una comparación de grupos (autoinculpación si/no). Para ello realizamos un análisis univariante. En el caso de las variables cuantitativas se obtuvieron medidas de centralización y dispersión y se estudió el grado de significación estadística mediante la t de Student. Para las variables cualitativas, se obtuvieron frecuencias y se utilizo la comparación de distribuciones según la prueba de Chi-cuadrado. Se obtuvo la matriz de correlaciones de Pearson para estudiar en la muestra global, la posible dependencia o independencia de las variables continuas. Finalmente se realizaron varios análisis multivariantes con regresión logística («Forward Stepwise LR / Adelante RV) en los que incluimos aquellas variables que, en el univariado, habían resultado significativas o estaban cercanas a serlo. Para valorar la capacidad discriminativa de estos resultados se realizó un analisis de Curva ROC. Los niveles de significación estadística utilizados para valorar las diferencias no atribuibles al azar, fueron los correspondientes a $p<0,05$.

\section{RESULTADOS}

\section{- Características sociodemográficas}

La edad media fue de 47,92 años (DT:13,19). El estado civil era el siguiente: casadas: $51,5 \%$; separadas/divorciadas: $29,9 \%$; solteras: $9 \%$ y viudas: $9,7 \%$. En cuanto a la situación laboral un $42,5 \%$ eran amas de casa; y un 38,8\% trabajaban fuera del hogar. El resto se encontraba sin empleo, en paro, jubiladas o en situación de incapacidad laboral. El nivel educativo era el siguiente: analfabetas: 1,5\%; estudios primarios: 36,6\%; Enseñanza.General.Básica:14,9\%; Bachillerato:17,2\%; Formación Profesional:17,9\%; Tituladas Medias: 8,2\%; Tituladas superiores:3,7\%

- Atribuciones de responsabilidad por la violencia en las mujeres maltratadas

Entre las mujeres que habían sufrido maltrato físico, se encontró que un 37,3\% pensaba que ellas habían sido fundamentalmente las responsables; un $47,6 \%$ pensaba que la responsabilidad era sobre todo de sus parejas y un $15,1 \%$ que era una responsabilidad compartida.

Posteriormente, preguntamos en todos los casos si consideraban que culpabilización era de tipo caracterial o conductual. Dentro de las mujeres que se autoinculparon, un $32,6 \%$ pensaban que la culpa era de tipo caracterial y un $67,4 \%$ conductual. 
Entre las mujeres que pensaron que la responsabilidad era fundamentalmente de sus parejas, un $67,7 \%$ pensaban que la culpa era caracterial y un $32,3 \%$ conductual. En los casos en que pensaron que la responsabilidad era de ambos (solo 19 mujeres), consideró caracterial su propia culpa en un $66,7 \%$ y la culpa de la pareja en un $59,1 \%$ de los casos. (figura 1 )

Figura 1

Subtipo de atribución frente al maltrato

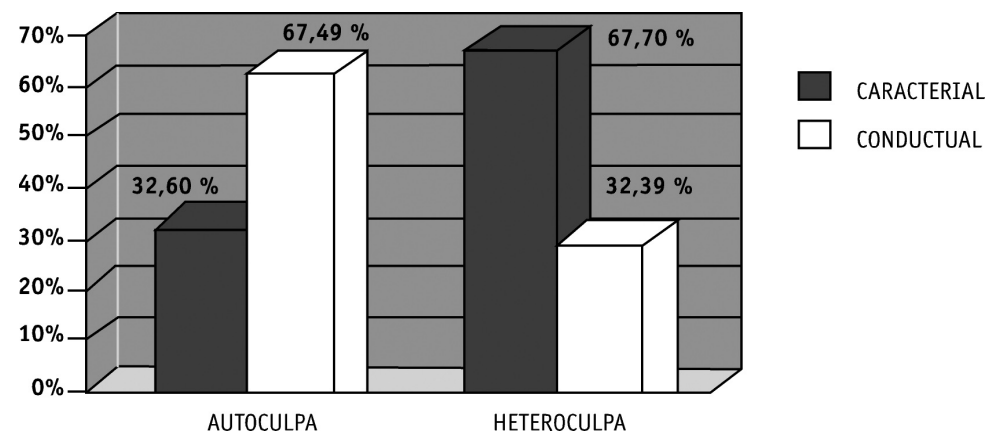

Para intentar estudiar si existía relación entre sentir culpabilidad y otras variables, clasificamos a las mujeres maltratadas en dos grupos: las que se habían sentido culpables y las que no se habían sentido (en este segundo grupo incluimos a las mujeres que pensaban que era su pareja la que era fundamentalmente responsable y las que pensaban que la responsabilidad era mixta; en este último grupo sólo se encontraban 19 mujeres). Encontramos las siguientes asociaciones (Tabla 1):

- Existió asociación entre haber sentido culpa por la violencia y tener antecedentes de separaciones en la infancia: en el grupo de mujeres que se autoculpabilizaron había un $45,7 \%$ con antecedentes de separaciones mientras que entre las que no lo hicieron la frecuencia de separaciones fue del $26,3 \%(p<0,05)$.

- Se encontró asociación entre autoinculpación y haber sufrido abuso físico en la infancia por parte del padre $(p<0,001)$. Así, en las mujeres que se culpabilizaron por la relación violenta de su pareja, la puntuación de agresión física en la infancia del padre según la escala CTS-P fue de 20,79 (DT: 31,16). En las que no lo hicieron, la puntuación en la misma escala fue de 6,25 (DT: 17,19). En relación con haber sufrido abuso sexual en la infancia, no se encontró relación entre esta variable y la autoinculpación.

- Observamos que las mujeres que se culpabilizaron tenían más antecedentes de intentos de suicidio que las que no. Así, en las primeras había un $45,7 \%$ de mujeres con intentos autolíticos, mientras que en el segundo grupo la frecuencia de éstos era del $13,8 \%(p<0,001)$. 
- Encontramos mayor frecuencia de mujeres que se culpabilizaban por la violencia en aquellas que dijeron conocer que su pareja había presenciado abusos físicos entre sus padres: un $46 \%$ de las mujeres de estas dijeron conocer que su marido había presenciado malos tratos entre sus padres, mientras que entre las mujeres que no se culparon, sólo un $27,5 \%$ dijeron conocer que su pareja había presenciado estos abusos $(p<0,05)$.

- También se encontró que se culpaban más las mujeres que dijeron conocer que su pareja había sufrido abuso físico en la infancia. Entre éstas, un 41,3\% informó que su pareja había sufrido abuso físico infantil; entre las que no se culparon por la violencia, sólo un $25 \%$ dijo que conocía este dato en su pareja $(p<0,05)$.

Tabla 1

Variables relacionadas con la autoinculpación

\begin{tabular}{|l|c|c|}
\hline & AUTOCULPA & NO AUTOCULPA \\
\hline Separaciones infantiles & $45.7 \%$ & $25 \%$ * \\
\hline Violencia del padre en la infancia & $20.79 \mathrm{CTS}$ & $6.25 \mathrm{CTS}$ ** \\
\hline Intentos autoliticos & $45.7 \%$ & $13.8 \%$ ** \\
\hline Violencia entre los padres de la pareja & $46 \%$ & $27.5 \%$ * \\
\hline Violencia a la pareja en la infancia & $41.3 \%$ & $26.3 \%$ * \\
\hline
\end{tabular}

${ }^{*} p<0,05$

${ }^{\star *} p<0,001$

No se observó relación entre el tipo de atribución y el diagnóstico que se realizó durante la entrevista clínica según criterios de la CIE-10 (OMS, 1992). Tampoco con haber sufrido consecuencias físicas al maltrato y con haber puesto denuncias. No se observó asociación entre la autoinculpación y la duración de la relación abusiva o la intensidad del abuso (medida por la escala CTS).

Subdividimos posteriormente atribuciones de autoinculpación y heteroinculpación en caracteriales y conductuales y estudiamos posibles relaciones con las mismas variables alternativas que usamos para el anterior análisis; sin embargo, no encontramos ninguna asociación significativa. Probablemente el escaso tamaño de la muestra contribuyó a esta falta de relación.

Predicción múltiple: En este análisis utilizamos las variables significativas o próximas a serlo, que presentaban las mujeres maltratadas que se culpaban del abuso de su pareja. En el análisis multivariado con regresión logística («Forward Stepwise LR / Adelante $\mathrm{RV}$ ») la única variable predictora fue haber sufrido maltrato del padre en la infancia.

El riesgo de autoinculpación es mayor en las mujeres con antecedentes de agresión física de su padre en la infancia, aumentando con una OR de 1.03 (IC 1.00 a 1.05) por cada unidad de incremento de la escala CTSP (Tabla 2). 
Tabla 2

Análisis multivariante de autoinculpación

\begin{tabular}{|l|c|c|c|c|c|}
\hline & COEFICIENTES & SIG. & EXP(B) & MINIM0* & MÁXIM0* \\
\hline Abuso físico del padre en la infancia &, 0262 & 0,0099 & 1,0265 & 1,0063 & 1,0472 \\
\hline Constante &,- 9422 & 0,0000 & & & \\
\hline
\end{tabular}

* Para un intervalo de confianza 95\%. Este modelo permite predecir el maltrato con un área bajo la curva ROC de 0.65 ( IC 0.54 a 0.76$)$.

\section{DISCUSIÓN}

En relación con el tipo de atribución que las mujeres maltratadas realizaron para ser objeto de violencia, en nuestro estudio observamos que un alto porcentaje de ellas se culpabilizaba de ser responsables de la violencia que su pareja había ejercido sobre ellas. Entre las que así lo hacían, el porcentaje de las que relacionaron esta culpa con su conducta fue mayor que las que lo relacionaron con su carácter. En el caso de las mujeres que culparon a su pareja hubo un mayor porcentaje que lo relacionaron con el carácter de él. Revisando estudios hechos sobre autoinculpación en mujeres maltratadas, se encuentran resultados diversos. 0'Leary (27) en una investigación realizada en un centro asistencias para víctimas de violencia doméstica, encontró que el $33 \%$ de las mujeres maltratadas de se culpaban de ser la causa de la violencia de sus parejas.

En nuestro estudio no encontramos relación entre la tendencia a la autoinculpación con la intensidad y la duración de la violencia en la relación. Sin embargo, revisando la literatura encontramos que hay varios estudios que si encuentran esta relación. Miller y Porter (28) a partir de sus datos de investigación en centros para víctimas de maltrato, sugieren que a medida que la violencia aumenta en severidad y los incidentes ocurren con mayor frecuencia, las mujeres dejan a autoinculparse. Los estudios de Frieze (29) también habían llegado a conclusiones parecidas. Encontraron en las mujeres de su investigación que tras un primer episodio de violencia, aparecían sentimientos de culpa en un 19\% ; sin embargo, en episodios repetidos, el porcentaje descendía a un $9 \%$. Quizá la ausencia de hallazgos positivos en nuestro estudio, en cuánto a relación entre autoinculpación con duración e intensidad de la violencia, puede deberse a que los estudios anteriores estaban hechos en mujeres que residían en centros para mujeres maltratadas. Este hecho probablemente suponga una intensidad y gravedad del problema y un grado de toma de conciencia del mismo que no presentan las mujeres de nuestro estudio. Recordemos que nuestro estudio se ha realizado en mujeres que acuden a un centro de salud mental por motivos no relacionados en principio con el hecho de haber sufrido maltrato. La frecuencia y la intensidad encontradas es notablemente inferior a las mujeres que están en centros 
residenciales específicos. Además, el hecho de que en nuestra muestra sea frecuente encontrar mujeres con síntomas depresivos puede ser un factor que influya en una mayor tendencia para atribuirse culpa.

Respecto a la interrelación entre la atribución de culpa y depresión, en nuestra investigación no estudiamos la relación entre intensidad de síntomas depresivos y tipo de atribución. Sin embargo, sí observamos que las mujeres que se autoinculpaban por la violencia habían tenido más intentos autolíticos que las que no lo hacian. Los estudios que hemos revisado sobre este tema muestran distintos hallazgos. En un estudio realizado en 33 mujeres maltratadas asistidas en un centro específico para violencia doméstica, se encontró que sólo el $12 \%$ se culpaban de la violencia de su pareja. No se observó relación entre esta autoinculpación con la duración del abuso ni con la frecuencia ni severidad de la agresión física. Sin embargo, si se halló relación con la sintomatología depresiva (22). Unos años antes, Walker (30) había asegurado que la autoinculpación que experimentaban las mujeres que habían sufrido maltrato les conducía a presentar clínica depresiva si la violencia persiste.

En un interesante estudio realizado por Andrews (27), en una muestra de 286 mujeres, se encontró que 72 de ellas habían sufrido violencia por parte de su pareja. De ellas, el $53 \%$ de las mujeres que habían sufrido maltrato se atribuían la culpa de ser víctima de una relación violenta. Al contrario de lo que ellos esperaban, hallaron que un $68 \%$ atribuía la culpa a aspectos relacionados con su comportamiento y conducta (culpa «conductual») y un 32\% a aspectos relacionados con su forma de ser (culpa «caracterológica»). Teniendo en cuenta el factor de haber sufrido abusos en la infancia, encontraron que no había diferencias en tasas de autoinculpación global entre personas que habían sufrido abuso y las que no lo habían sufrido. Sin embargo, hallaron que en el grupo de mujeres que habían experimentado abuso en la infancia era más frecuente observar la atribución caracterial. Se encontró la relación entre culpa y depresión dependía del tipo de convivencia con la pareja. Entre mujeres que habían abandonado a su pareja, las que todavía se culpabilizaban de la relación violenta estaban significativamente más deprimidas que las que culpaban a su pareja. Sin embargo, no se encontró esta asociacion entre las mujeres que vivían todavía con su pareja. Comparando este estudio con el nuestro, observamos que la tendencia a la autoinculpación es mayor en este trabajo (53\% de mujeres se autoinculpaban frente a un $37 \%$ en nuestro estudio). Hay que destacar que la metodología para valorar el tipo de atribución para la violencia fue similar en los dos estudios. Dentro de las mujeres que se culpaban, los porcentajes de mujeres que se atribuían culpa conductual fueron similares en ambos estudios. En los dos casos hubo mayor porcentaje de mujeres que se atribuyeron culpa conductual que caracterial.

Con relación a la autoinculpación de las mujeres y datos de sus parejas, en nuestro estudio observamos asociación entre autoinculpación y el hecho de que las mujeres conocieran que sus parejas habían sufrido abuso físico en la infancia por parte de 
sus padres y hubieran presenciado violencia entre ellos. Estos dos últimos hallazgos pensamos que podrían relacionarse con la justificación que, de alguna manera, hace la mujer que sufre maltrato a su pareja al considerarlo como «víctima» de una situación anterior. No hemos encontrado estudios que nos sirvan para comparar estos datos. Quizá el modo tradicional de socialización de la mujer provoca que, en muchas ocasiones, al unirse con una pareja con un pasado conflictivo, tienda a pensar que puede «redimirle» de esa situación. La observación en la mujer de que, a pesar de sus intentos por «salvar» a su pareja y la relación con él, el resultado es una relación con violencia, pensamos que puede provocar en ella el sentimiento de que ella es responsable, en mayor o menor medida, de no haber sido capaz de cambiar a su pareja ni a su relación.

El único factor que figuró en análisis multivariante, como variable predictora de que la mujer se atribuyera autoinculpación por el abuso, fue haber sufrido abuso físico de su padre en la infancia. Esta idea confirma nuestra hipótesis de que haber sufrido abuso infantil supone un factor de riesgo para permanecer en la vida adulta en relaciones de abuso y atribuirse autoinculpación por la existencia del mismo. Como ya hemos comentado, pensamos que la experiencia de abuso en la infancia provoca en el niño un mecanismo de confusión. Por el hecho de experimentar amor hacia el agresor, puede no registrarse el perjuicio o puede disculparse permanentemente al que daña. Lo primero que puede hacer el niño para sobrevivir es intentar justificar el maltrato. Para ello, puede intentar buscar una explicación que asigne sentido a esa acción de la que es víctima. Pero como esa acción fue contra él, puede llegar a suponer que su propia persona y sus propias conductas son negativas y sentirse culpable de la agresión. Este mecanismo puede persistir en la edad adulta y producir que se comprendan y se justifiquen las acciones de los demás, y una vez más, las víctimas vuelvan a sentirse culpables de la agresión. Queda pendiente para otros estudios, investigar por qué esta relación entre agresión física en la infancia y maltrato en la relación de pareja, se produce sólo si la agresión ha sido del padre.

Pensamos que nuestro estudio ayuda a confirmar la idea de que determinados patrones relacionales y experiencias infantiles constituyen factores que contribuyen a que en la edad adulta se produzcan relaciones de pareja en las que exista maltrato y a que las mujeres se culpabilicen por el mismo. Como limitaciones a nuestro trabajo somos conscientes de que nuestros datos se centran en una muestra de pacientes que acuden a un centro de salud mental, por lo que los resultados no son extrapolables a otros sectores. Pensamos que sería interesante realizar estudios similares en otros grupos de población. Por otra parte, la complejidad de la medida de la atribución con metodología cuantitativa, nos sugiere que resultaría de interés introducir estudios cualitativos que nos permitieran estudiar estos fenómenos desde otras perspectivas. 


\section{BIBLIOGRAFÍA}

1. Vázquez, B. Evaluación psicológica de la mujer maltratada. Clínica y Salud, 199; 10(3):377-391.

2. Villavicencio,P. Mujeres maltratadas: Conceptualización y evaluación. Clínica y Salud 1993;4(3),215-228.

3. Lorente Acosta M., Lorente Acosta J.A. Agresión a la mujer: maltrato, violación y acoso. Granada, Comares, 1999

4. Abramson L.Y., Seligman M.E.P., Teasdale J. Learned helplessness in humans: Critique and reformulation. Journal of Abnormal Psychology 1978; 87:49-74.

5. Ickes W.J., Layden M.A. Attributional styles. En: J.H.Harvey, W.J. Ickes and R. F. Kidd (Ed). New directions in attribution research (vol 2) Erlbaum. NY, Hillsdale, 1978.

6. Brewin C.R. Attributions for industrial accidents: Their relationship to rehabilitation outcome. Journal of Social and Clinical Psychology 1984; 2:156-164.

7. Bulman R.J., Wortman C.B. Attributions of blame and coping in the «real world»: Severe accident victims react to their lot. Journal of Personality and Social Psychology 1977; 35: 351-365.

8. Janoff-Bulman R. Characterological versus behavioral self-blame: Inquiries into depression and rape. Journal of Personality and Social Psychology 1979; 37, 10:1798-1809.

9. Andrews B., Brewin C.R. Attributions of blame for marital violence: a study of antecedents and consequences. Journal of Marriage and the Family 1990;52:757-767.

10. Miller D.T., Porter C.A. Self-blame in victims of violence. Journal of Social Issues 1983; 39(2): 139-152.

11. Cascardi M., 0'Leary K.D. Depressive symptomatology, self-esteem, and self-blame in battered woman. Journal of Family Violence 1992; 7(4)249-259.

12. Eisikovits Z., Enos G. Awareness of guilt and shame in intimate violence. Violence and Victims 1997; 12(4):307-322.

13. Messman T.L. , Long P.J. Child sexual abuse and its relationship to revictimization in adult women: a review. Clinical Psychology Review 1996; 16:397-420.

14. Briere J., Woo R., MC Rae B., Foltz J., Sitzman R. Lifetime victimization history, demographics and clinical status in female psychiatric emergency room patients. Jounal of Nervous and Mental Disease 1997; 185:95-101.

15. Arata C.M., Lindman L. Marriage, child abuse and sexual revictimization. Journal of Interpersonal Volence 2002; 17:953-971.

16. Chu J.A., Dill D.L: Dissociative symptoms in relation to childhood physical and sexual abuse. American Journal of Psychiatry 1990; 147:887-892.

17. Kessler B.L., Bieschke K.J. A retrospective analysis of shame, dissociation and adult victimization in survivors of childhood sexual abuse. Journal of Counseling Psychology 1999; 46:355-341. 
18. Koss M.P., Dinero T.E. Discriminant analysis of risk factors for sexual victimization among a national sample of college women. Journal of Consulting and Clinical Psychology 1989;57:242-250.

19. Merrill L.L., Newell C.F., Thomsen C.J., Gold S.R., Milner J.S., Koss M.P. Childhood abuse and sexual revictimization in a female navy recruit sample. Journal of Traumatic Stress 1999; 12:211-225.

20. Messman-Moore T.L., Long P.J. Child sexual abuse and revictimization in the form of adult sexual abuse, adult physical abuse and adult psychological maltreatment. Journal of Interpersonal Violence 2000;15:289-502.

21. Ravazzola M.C. Historias infames: los maltratos en las relaciones. Buenos Aires, Paidós Terapia Familiar, 1997.

22. Cascardi, M., O'Leary, K.D. Depressive symptomatology, self-esteem, and selfblame in battered woman. Journal of Family Violence 1992;7(4): 249-259.

23. Straus M.A. Measuring intrafamily conflict and violence: The conflict tactics scales. Journal of Marriage and the Family 1979; 41:75-88.

24. Straus M.A., Smith C. Violence in Hispanic families in the United states: incidence rates and structural interpretationos. En: M.A. Straus.(Ed.). Physical violence in American families: Risk factors and adaptations to violence in 8.145 families. New York, Transaction Publishers, 1990.

25. Straus M.A. Handbook of Conflict Tactics Scale(CTS). Family Research Laboratory. University of New Hampshire, Durham, 1999.

26. Finkelhor D. Sexually victimized children. New York, Free Press., 1979.

27. O'Leary D.K., Curley A., Rosenbaum A., Clarke C. Assertion training for abused wives: A potentially hazardous treatment. Journal Marital Family Therapy 1985; 11:319-322.

28. Miller, D.T., Porter, C. ASelf-blame in victims of violence. Journal of Social Issues 1983; 39(2): 139-152

29. Frieze I. Perceptions of battered wives. En: I.H. Frieze, D.Bar-Tal and J.S.Carroll (Ed.). New approaches to social problems: applications of attribution theory. San Francisco, Jossey-Bass, 1979.

30. Walker L.E. The battered women. New York, Harper and Row, 1979.

Cristina Polo Usaola

Psiquiatra

Centro de Salud Mental Hortaleza. Madrid

Marisa López Gironés

Psicóloga

Centro Salud Mental de Hortaleza. Madrid 
Daniel Olivares Zarco

Psiquiatra

Hospital Universitario de Guadalajara

Antonio Escudero Nafs

Psiquiatra

Centro de Salud Mental Majadahonda. Madrid

Beatriz Rodríguez Vega

Psiquiatra

Hospital Universitario La Paz. Madrid

Alberto Fernández Liria

Psiquiatra

Hospital Universitario Príncipe de Asturias. Alcalá. Madrid

Dirección correspondencia:

Cristina Polo Usaola

Centro de Salud Mental de Hortaleza

C/. Mar Caspio $n^{\circ} 8$

Madrid

E-mail: cristinapolo83@hotmail.com 